Series A

I. MATHEMATICA

507

\title{
BOUNDARY BEHAVIOR OF QUASIREGULAR MAPPINGS
}

BY

O. MARTIO and S. RICKMAN

H E L S N K I 1972

S U O M A L A I EN TIEDEAKATEMIA

doi:10.5186/aasfm.1972.507 
Copyright (C) 1972 by Academia Scientiarum Fennica ISBN $951-41-0034-4$

Communicated 14 January 1972 by JUSSI VäIs̈̈LÄ 


\section{Introduction}

Quasiregular mappings can be regarded as an $n$-dimensional generalization of the analytic functions in plane. For the theory of these mappings we refer to $[4-6]$. Compared to the thorough and extensive studies made on the boundary behavior of analytic functions (see $[3 ; 7]$ ) very little has been written so far on the boundary behavior of quasiregular mappings for dimensions $n \geq 3$. However, some results proved earlier deserve mentioning. In $[5,4.1]$ it was proved that a set of capacity zero is removable for a quasiregular mapping provided that the mapping omits a set of positive capacity. This is known as the theorem of af Hällström for analytic functions. In $[6,3.14-3.18]$ some relations for asymptotic values, exceptional values, and the branch set were considered in the case of an isolated essential singularity. A proof for an $n$-dimensional rersion of Iversen's theorem for an isolated singularity was also indicated [6,3.18]. It has also been proved that an isolated boundary point is always removable if the mapping is a local homeomorphism $[1 ; 15 ; 16]$.

In the plane a quasiregular mapping can be represented as a composition of a quasiconformal (homeomorphic) mapping and an analytic function. Hence the problem for dimension $n=2$ reduces to the study of boundary behavior of analytic functions and quasiconformal mappings. Since a quasiconformal mapping of a disc onto itself need not preserve sets of measure zero on the boundary. sereral classical theorems for analytic functions of a disc, among others Fatou's theorem on the existence of radial limits, fail to hold for quasiregular mappings in the case $n=2$; see for example [7, pp. $119-120 ; 11]$. At present there seems to be no reason why for example Fatou's theorem should be true for quasiregular mappings in dimensions $n \geq 3$.

In this paper we shall give further results which in general are new only for dimensions $n \geq 3$ although some proofs simplify also the known proofs for $n=2$. We start by proving a relation for cluster sets and boundary cluster sets at a boundary point known as Iversen-Tsuji's theorem in the case $n=2$. The next section is devoted to the study of asymptotic values and we state two results proved for $n=2$ by Noshiro [7] and Cartwright [2]. The rest of the paper deals with mappings of a ball. 
We prove the reflection principle and discuss the existence of radial, angular, and asymptotic limits. We close by considering quasiregular mappings whose increase of the multiplicity is bounded by specific functions when approaching the boundary. Two classical theorems in the theory of analytic functions concerning radial limits are presented for these mappings, namely, the theorem of Fatou and the theorem of F. and M. Riesz.

\section{Notation and preliminaries}

2.1. We shall recall some of the notation and terminology of [4-6] which will be the base also here. In addition, some further notions will be introduced. The notation $f: G \rightarrow R^{n}$ or $f: G \rightarrow \bar{R}^{n}$ includes the assumptions that $G$ is a domain in the euclidean $n$-space $R^{n}$ or in the compactified $n$-space $\bar{R}^{n}=R^{n} \cup\{\infty\}$, respectively, and that $f$ is continuous. All topological operations are performed with respect to $\bar{R}^{n}$. Throughout the paper we assume that $n \geq 2$. If $A$ is a segment of line and if $\alpha: \Delta \rightarrow \bar{R}^{n}$ is a path, we let $x$ denote the locus $x \Delta$ of $\alpha$. If $y \in R^{n}$, we denote by $\gamma_{y}$ the path $\gamma_{y}:[0,1] \rightarrow R^{n}$ defined by $\gamma_{y}(t)=t y$. The inner product of $x, y \in R^{n}$ is denoted by $(x y)$.

2.2. Cluster and asymptotic sets. For a mapping $f: G \rightarrow \bar{R}^{n}$ and a set $F \subset \partial G$ we set

$$
C(f, F)=\bigcup_{y \in F} C(f, y)
$$

where the cluster set of $f$ at $y$ is

$$
C(f, y)=\bigcap_{L^{-}} \overline{f\left(G \cap C^{\top}\right)}
$$

where $U$ runs over all neighborhoods of $y$. We shall also employ the boundary cluster set of $f$ at $y \in \partial G$ with respect to $F$ defined as

$$
C_{F}(f, y)=\bigcap_{U} \overline{C(f, L \cap F \backslash\{y\})}
$$

where $U$ runs over all neighborhoods of $y$.

A point $z \in \bar{R}^{n}$ is an asymptotic value of $f$ at a boundary point $y \in \partial G$ if there exists a path $\gamma:[0,1) \rightarrow G$ with $\lim _{t \rightarrow 1} \gamma(t)=y$ and $\lim _{t \rightarrow 1} f(\gamma(t))=z$. The asymptotic set $A(f, y)$ of $f$ at $y$ is the set of asrmptotic values of $f$ at $y$.

2.3. Capacities. In $[4,5.4]$ we defined the capacity of a condenser (see also $[5$, p. 4]) and in $[5,2.12]$ the concept of a set of capacity zero. 
By capacity in these definitions and in this paper we always mean conformal capacity. In connection with studies of analytic functions in the plane the potential theoretic capacity is most commonly used $[3 ; 7]$. However, for $n=2$ a set is of conformal capacity zero if and only if it is of potential theoretic capacity zero. This fact and other relations between these two concepts of capacities are proved in [12].

2.4. Moduli of path families under quasiregular mappings. The main tools in our proofs are two inequalities for the moduli of path families. Let $f: G \rightarrow R^{n}$ be a quasiregular mapping $[4,2.20]$. The outer dilatation inequality

$$
M(\Gamma) \leq N(f, A) K_{o}(f) M(f \Gamma)
$$

was proved in $[4,3.2]$. Here $\Gamma$ is a family of paths in a Borel set $A \subset G$, $M(\Gamma)$ its $n$-modulus, $K_{o}(f)$ the outer dilatation of $f[4,2.20]$, and $N(f, A)$ is the supremum of the number (possibly $x$ ) of points in $A \cap f^{-1}(y)$. This inequality has a drawback as it contains the multiplicity which in general tends to infinity as the path family approaches the boundary. Because of this, (2.5) is used only in the proofs of two last theorems.

The second, the inner dilatation inequality

$$
M(f \Gamma) \leq K_{I}(f) M(T),
$$

which was proved by Poleckii [8], is of more use in general boundary problems. Here $K_{I}(f)$ is the inner dilatation of $f[4,2.20]$ and $T$ a family of paths in $G$.

\section{A relation for cluster sets}

3.1. Our first result is a theorem on the behavior of the norm of a quasimeromorphic mapping $[5,2.1]$ when approaching a boundary point from the domain and along the boundary. First we need the following lemma which is a path family version of $[5,2.14]$.

3.2. Lemma. Let $D \subset R^{n}$ be a bounded domain, let $C$ be a compact set in $D$, and let $F \subset D \backslash C$ be closed in $D$ with cap $F=0$. Let further $\Gamma_{1}\left(\right.$ resp. $\left.\Gamma_{2}\right)$ be the family of paths joining $\partial D$ (resp. $\left.\partial D \cup F\right)$ and $C$ in $D$. Then $M\left(\Gamma_{\mathrm{j}}\right)=M\left(\Gamma_{2}\right)$.

Proof. We apply $[5,2.14]$ to the condenser $(\mathrm{D}, \mathrm{C})$ and to the set $F$. This gives c.ip $(D \backslash F, C)=\operatorname{cap}(D, C)$. The lemma follows from [14, Theorem 38 . 
3.3. Theorem. Let $f: G \rightarrow \bar{R}^{n}$ be a quasimeromorphic mapping, let $E \subset \partial G$ be a compact set of capacity zero, let $y \in E \cap \overline{(\partial G \backslash E)}$, and let $f(G \cap U)$ be bounded for some neighborhood $U$ of $y$. Then

$$
\limsup _{x \rightarrow y}|f(x)|=\limsup _{\substack{z \rightarrow y \\ z \in \partial G \backslash E}}\left(\limsup \sup _{x \rightarrow z}|f(x)|\right) .
$$

Proof. We denote by $a$ and $b$ the left and right hand sides of the inequality (3.4) respectively. The inequality $a \geq b$ is trivial. Suppose $a>b$ and let $0<\varepsilon<a-b$. We may assume $y=0$. We choose $\varrho_{1} \in$ $(0,1 / 2)$ such that $B^{n}\left(2 \varrho_{1}\right) \subset U^{\prime}$ and

$$
\limsup _{x \rightarrow \pm} f(x) \mid<b-\varepsilon
$$

whenever $z \in B^{n}\left(2 \varrho_{1}\right) \cap(\partial G \backslash E)$. For $k=1,2, \ldots$ set

$$
\begin{gathered}
\varrho_{k+1}=\varrho_{1} / 2^{k}, \\
M_{k}=\sup \left\{f(x) \| x \in B^{n}\left(2 \varrho_{k}\right) \cap G\right\} .
\end{gathered}
$$

Let $x \in B^{n}\left(\varrho_{1}\right) \cap G$ and let $B^{n}(x, \delta) \subset B^{n}\left(\varrho_{1}\right) \cap G$ for some $\delta>0$. Since $y \in \overline{\partial G \backslash E}$ and since $E$ is compact, there exists a point $x^{\prime} \in B^{n}\left(\varrho_{1}\right) \cap$ $(\partial G \backslash E)$ such that $B^{n}\left(x^{\prime} . \delta^{\prime}\right) \cap E=\varnothing$ for some $\delta^{\prime}, 0<\delta^{\prime}<Q_{1}-x^{\prime} \mid$. Since $E$ is of capacity zero, there is a segment of line in $\mathbf{C} E$ with endpoints in $B^{n}(x, \delta)$ and $B^{n}\left(x^{\prime}, \delta^{\prime}\right)$ [5, p. 8]. Hence there exists a path $l:[0,1] \rightarrow B^{n}\left(\varrho_{1}\right) \cap \mathbf{C} E$ with $l(0)=x$ and $l(1)=x^{\prime}$. Lat $t \in(0,1]$ be such that $A=l[0, t) \subset G$ and $l(t) \in \partial G$. Denote by $G_{1}$ the $x$-component of $B^{n}\left(2 \varrho_{1}\right) \cap G$.

Assume $f(x)>b+\varepsilon$. We denote by $T$ the family of paths $\gamma$ : $[0,1] \rightarrow \bar{B}^{n}\left(M I_{1}\right) \backslash B^{n}(b-\varepsilon)$ for which $\gamma(0) \in f A$ and $\gamma(1) \in S^{n-1}\left(M_{1}\right)$. Let $\tilde{\Gamma}$ be the family of maximal liftings with respect to the mapping $f \mid G_{1}$ of the paths in $T$ starting at some point in $A[6,3.12]$. Then every path in $\tilde{\Gamma}$ either is unrectifiable or belongs to the set $\tilde{\Gamma}_{1}$ of rectifiable paths in $\tilde{\Gamma}$ and ends in $\left(S^{n-1}\left(\underline{\bullet}_{1}\right) \cap \bar{G}\right) \cup E$.

Let now $\hat{\Gamma}$ be the family of paths in $B^{n}\left(2 \varrho_{1}\right)$ joining $\bar{A}$ and $S^{n-1}\left(2 \varrho_{1}\right) \cup E$. Then

$$
M(\hat{\Gamma}) \geq M\left(\tilde{\Gamma}_{1}\right)=M(\tilde{\Gamma})
$$

by 3.2 and we have

$$
M(\hat{\Gamma}) \leq \omega_{n-1}(\log 2)^{1-n}
$$

by $[13,7.5]$. On the other hand, every sphere $S^{n-1}(u, s)$ meets both $f A$ 
and $S^{n-1}\left(M_{1}\right)$ where $u=M_{1} f(x) /|f(x)|$ and $M_{1}-|f(x)|<s<M_{1}-(b+\varepsilon)$. It follows by [13, Theorem 10.12] that

$$
M(\Gamma) \geq c_{n} \log \frac{M_{1}-(b+\varepsilon)}{M_{1}-f(x) \mid}
$$

where $c_{n}>0$ is a constant which depends only on $n$. By $[6,3.12]$ the family $T$ is minorized by $f \tilde{T}$, hence $M(f \tilde{T}) \geq M(T)$. Combining this with (2.6), (3.5), (3.6), and (3.7) we obtain an inequality

$$
\frac{M_{1}-|f(x)|}{M_{1}-(b+\varepsilon)} \geq p>0
$$

where $\beta<1$ depends only on $n$ and $K_{I}(f)$. This yields an estimate

$$
M_{2} \leq b+\varepsilon+(1-\beta)\left(M_{1}-(b+\varepsilon)\right) \text {. }
$$

Proceeding successively we get

$$
M_{k} \leq b+\varepsilon+(1-\beta)^{k-1}\left(M_{1}-(b+\varepsilon)\right) .
$$

But $a \leq M_{k}$ for every $k$ and we have thus $a \leq b+\varepsilon$ by (3.8) which is a contradiction in view of the choise of $\varepsilon$. The theorem is proved.

As a corollary one obtains the following extension of the maximum principle.

3.9. Corollary. Let $f: G \rightarrow R^{n}$ be a quasiregular mapping of a bounded domain $G$ and let $E \subset \partial G$ be a compact set of capacity zero. If $\lim \sup \mid f(x)$ $\leq M$ for every point $y \in \partial G \backslash E$ and if for every $z \in E \stackrel{x \rightarrow y}{f(U \cap \cap G)}$ is bounded for some neighborhood $U$ of $i$, then $f \leq M$.

Proof. It follows from the assumptions that $f(I \cap G)$ is bounded for some neighborhood $V$ of $\partial G_{t}$. Hence $f$ is bounded. Set $F=\overline{\partial G \backslash E}$. $G_{1}=G \cup(E \backslash F)$, and $E_{1}=E \cap F$. since $E$ is of capacity zero and since $G$ is bounded, $F \neq O$ and $G_{r_{1}}$ is a domain with $\partial G_{1}=F$. Furthermore, $\partial G \backslash E=\partial G_{1} \backslash E_{1}$. By $[5,1.11]$ there exists an extension of $f$ to a quasiregular mapping $f_{1}: G_{1} \rightarrow P^{n}$ such that for $x \in G_{1}$

$$
f_{1}(x)=\lim _{u \rightarrow t} f(u) \text {. }
$$

Hence $f_{1}$ is also bounded and for $y \in \partial G_{1} \backslash E_{1}$

$$
\lim \sup _{x \rightarrow y}\left|f_{1}(x)\right|=\lim \sup _{x \rightarrow y} \mid f(x) \leq \leq M .
$$


If $y \in E_{1} \cap \partial G_{1}=E_{1} \cap \overline{\left(\partial G_{1} \backslash E_{1}\right)}$, then Theorem 3.3 applied to $f_{1}, E_{1} \subset \partial G_{1}$ : and $y$ gives

$$
\limsup _{x \rightarrow y}\left|f_{1}(x)\right| \leq M .
$$

Therefore $\left|f_{1}\right|$ and hence $|f|$ are bounded by $M$.

Theorem 3.3 can also be stated in the following form by means of the cluster set and the boundary cluster set. A proof based on 3.3 can be given analoguously to the case $n=2$. see [7. p. 17].

3.10. Theorem. Let $f: G \rightarrow \bar{R}^{n}$ be a quasimeromorphic mapping, let $E \subset \partial G$ be a compact set of capacity zero, and let $y \in E \overline{E(\partial G \backslash E)}$. Then $\partial C(f, y) \subset \partial C_{\partial G \backslash E}(f, y)$.

3.11. Remark. Theorem 3.3 or its equivalent form 3.10 is known for $n=2$ as Iversen-Tsuji's theorem. The given proof here simplifies the known proofs for the case $n=2$, see [7, pp. 17-19].

\section{Asymptotic values.}

4.1. In this section we shall prove some extensions of the $n$-dimensional version of Iversen's theorem [6, 3.18]. The study of asymptotic values will be continued by Theorem 5.11 in the next section. The following result was proved for meromorphic functions in the plane by Noshiro $[7$, p. 14].

4.2. Theorem. Let $f: G \rightarrow \bar{R}^{n}$ be a quasimeromorphic mapping, let $E \subset \partial G$ be a compact set of capacity zero, and let $y \in E$. If $z \in(C(f, y)$ $\left.C_{\partial G \backslash E}(f, y)\right) \cap \mathbf{C} f(G \cap U)$ for some neighborhood $U$ of $y$, there exists a sequence $x_{1}, x_{2}, \ldots$ of points in $E$ converging to $y$ such that $z \in A\left(f, x_{k}\right)$ for $k=1,2, \ldots$.

Proof. Let $z \in\left(C(f, y) \backslash C_{\partial G \backslash E}(f, y)\right) \cap \mathbf{C} f(G \cap U)$ for some neighborhood $U$ of $y$. We may assume $y=z=0$. There exists a sequence $r_{1}, r_{2}, \ldots$ of positive numbers tending to 0 such that $\bar{B}^{n}\left(r_{k}\right) \subset U$, $z \notin F=\overline{C\left(f,(\partial G \backslash E) \cap \bar{B}^{n}\left(r_{k}\right)\right)}$, and $S^{n-1}\left(r_{k}\right) \cap E=\varnothing$ for $k=1,2, \ldots$ This is possible because $A_{1}(E)=0$; see the proof of $[6,3.1]$. Fix $k$. There exists $\varrho_{k}>0$ such that $B^{n}\left(\varrho_{k}\right) \cap\left(F \cup f\left(S^{n-1}\left(r_{k}\right) \cap G\right)\right)=\varnothing \quad$ for otherwise there is a sequence $u_{1}, u_{2}, \ldots$ of points in $S^{n-1}\left(r_{k}\right) \cap G$ converging to a point of $(\partial G \backslash E) \cap S^{n-1}\left(r_{k}\right)$ and with $f\left(u_{i}\right) \rightarrow z$ as $i \rightarrow \propto$ which is impossible. 
Since $z \in C(f, y)$, there exists $x \in G \cap B^{n}\left(r_{k}\right)$ with $f(x) \in B^{n}\left(\varrho_{k}\right)$. By $[4,2.6]$ and $[4,2.9]$ there exists a closed $(n-1)$-dimensional cap $C \subset$ $S^{n-1}(|f(x)|) \cap f\left(G \cap B^{n}\left(r_{k}\right)\right)$ such that $f(x) \in C$, the $x$-component $\tau^{y}$ of $f^{-1} C$ is contained in $B^{n}\left(r_{k}\right)$, and such that $f$ maps $\tilde{C}$ surjectively onto $C$. Let $\Gamma$ be the family of paths $\gamma_{y}, y \in C$, and let $\tilde{\Gamma}$ be the family of maximal liftings of the paths in $\Gamma$ terminating in $\tilde{C}$. Let $\tilde{\gamma}:(t, 1] \rightarrow R^{n}$ be a path in $\tilde{\Gamma}$. We claim that there exists the limit

$$
\lim _{u \rightarrow t} \tilde{\gamma}(u)
$$

and it lies in $E \cap B^{n}\left(r_{k}\right)$. Since $B^{n}\left(\varrho_{k}\right) \cap f\left(S^{n-1}\left(r_{k}\right) \cap G\right)=\varnothing, \tilde{\gamma} \mid \subset B^{n}\left(r_{k}\right)$. If the limit (4.3) exists, it lies in $\partial G \cap \bar{B}^{n}\left(r_{k}\right)$ because $0 \in \mathbf{C} f\left(G \cap \bar{B}^{n}\left(r_{k}\right)\right)$. There can be no limit (4.3) which lies in $(\partial G \backslash E) \cap \bar{B}^{n}\left(r_{k}\right)$ because $B^{n}\left(\varrho_{k}\right) \cap F=\varnothing$. If the limit (4.3) does not exist, there is a sequence $\left(u_{i}\right)$ of points in $(t, 1)$ such that $u_{2 j}>u_{2 j+1}>u_{2 j-2}$ for $j=1,2, \ldots$ and that $a=\lim _{j \rightarrow \infty} \tilde{\gamma}\left(u_{2 j}\right)=b=\lim _{j \rightarrow \infty} \tilde{\gamma}\left(u_{2 j+1}\right)$. Set $A_{j}=\tilde{\gamma}\left[u_{2 j \div 1} \cdot u_{2 j}\right]$. Then $A=\lim \sup A_{j}$ is a connected set with $a, b \in \bar{A}$ which lies in $\partial G \cap \bar{B}^{n}\left(r_{k}\right)$ by $[6,3.12]$. Since $E$ is of capacity zero, there exists a sequence $\left(v_{j}\right)$ such that $u_{2 j}>v_{j}>u_{2 j+1}$ and $\lim \tilde{\gamma}\left(v_{j}\right) \in(\partial G \backslash E) \cap \bar{B}^{n}\left(r_{k}\right)$, but this is again impossible because $F \cap \stackrel{j \rightarrow \infty}{ } F B^{n}\left(\varrho_{k}\right)=\varnothing$. Hence the limit (4.3) exists and belongs to $E \cap B^{n}\left(r_{k}\right)$. Lemma 3.2 gives $M(\tilde{\Gamma})=0$ and by $(2.6)$ we have $M(f \tilde{\Gamma})=0$. But this means that almost every path $f \circ \tilde{\gamma}$, $\tilde{\gamma} \in \tilde{\Gamma}$, has the origin as one endpoint. This shows that $0 \in A\left(f, x_{k}\right)$ for some $x_{k} \in E \cap B^{n}\left(r_{k}\right)$. The theorem is proved.

As a corollary we obtain the following result which for meromorphic functions in the plane is known as Cartwright's theorem.

4.4. Corollary. Ler $G \subset \bar{R}^{n}$ be a domain and let $E \subset G$ be a set of capacity zero and closed in $G$. Let $f: G \backslash E \rightarrow \bar{R}^{n}$ be a quasimeromorphic mapping with nondegenerate $C(f, y)$ for every $y \in E$. If $y_{0} \in E$ and if $z \in \mathbf{C} f((G \backslash E) \cap U)$ for some neighborhood $L$ of $y_{0}$, there exists a sequence $x_{1}, x_{2}, \ldots$ of points in $E$ converging to $y_{0}$ such that $z \in A\left(f, x_{k}\right)$ for $k=1,2 \ldots$.

Proof. Suppose $y_{0} \in E$ and $z \in \mathbf{C} f\left((G \backslash E) \cap C^{\prime}\right)$ for some neighborhood $U$ of $y_{0}$. We may assume that $y_{0} \propto$. If $\operatorname{cap} \mathbf{C} f\left(B^{n}\left(y_{0}, r\right) \backslash E\right)$ $>0$ for some $r$ with $0<r<d\left(y_{0}, \partial G\right), f B^{n}\left(y_{0}, r\right) \backslash E$ can be extended to a quasimeromorphic mapping of $B^{n}\left(y_{0}, r\right)$ by [5, Theorem 4.1] and $C\left(f, y_{0}\right)$ is degenerate contrary to the assumption. Hence $z \in C\left(f, y_{0}\right)$ $=\bar{R}^{n}$. The assertion follows from 4.2 because $\partial(G \backslash E) \backslash E=\partial G$ and $C_{\partial G}\left(f, y_{0}\right)=\varnothing$. 


\section{Mappings of a ball}

5.1. In this section we study quasiregular mappings of a ball. We start by the reflection principle. After a brief discussion on radial and angular limits we show that asymptotic limits exist in a dense set if the mapping omits a set of positive capacity. We close the section by a study of quasiregular mappings with some conditons on the increase of the multiplicity when approaching the boundary.

We state the reflection principle in the following form.

5.2. Theorem. (Reflection principle). Let $G$ be a domain in the unit ball $B^{n}$ and let $A \subset \partial G \cap S^{n-1}$ be non-empty such that for every $y \in A$ there exists a neighborhood $U$ for which $U \cap \partial G=U \cap A$. Let $f: G \rightarrow B^{n}$ be a quasiregular mapping with $C(f, \partial G) \subset \partial f G$ and $C(f, A) \subset S^{n-1}$. Then there is a quasimeromorphic mapping $g: G \cup A \cup_{\alpha} G \rightarrow \bar{R}^{n}$ such that $g\left(G=f\right.$ and $K(g)=K(f)$ where $\alpha$ is the reflection in $S^{n-1}$.

Proof. We first show that the multiplicity $N(f, G)$ is finite. Fix a point $u$ in $f G$. Since $C(f, \partial G) \subset \partial f G$, there exists a domain $E_{0}$ with $\bar{E}_{0} \subset G$ such that $f^{-1}(u) \subset E_{0}$. Then (see [4, p. 11])

$$
N(u, f . G) \leq \mu\left(u, f, E_{0}\right)=\sum_{x \in f^{-1}(u)} i(x, f)=\mu(u, f, E)
$$

for any domain $E \supset E_{0}$ with $\bar{E} \subset G$. If $v$ is any point in $f G$, we can by the assumption $C(f, \partial G) \subset \partial f G$ choose $E$ such that $u$ and $v$ are in the same component of $f E \backslash f \partial E$ and such that $f^{-1}(v) \subset E$. Then

$$
N(v, f, G) \leq \mu(v, f, E)=\mu(u, f, E)=\mu\left(u, f, E_{0}\right) .
$$

Hence $N(f, G) \leq u\left(u, f, E_{0}\right)<\infty$.

Let now $\phi: \bar{R}^{n} \rightarrow \bar{R}^{n}$ be a Möbius transformation which maps $B^{n}$ onto the half space $H^{n}=\left\{x \in R^{n}: x_{n}>0\right\}$. Set $\psi=f \circ \psi^{-1} \psi G$. We next show that

$$
\lim _{t \rightarrow 0} \psi\left(u+t e_{n}\right)
$$

exists for almost every point $u$ in $\varphi A$. It suffices to show this in a neighborhood of every finite point of $\varphi A$. Let therefore $y \in q A$ be finite. By assumption there is $\delta>0$ such that $C_{1}=\bar{C} \cap H_{-}^{n} \subset q G$ and $F=$ $\left\{u \in \bar{C} \mid u_{n}=0\right\} \subset_{\varphi} A$ where $C$ is the cube $\left\{x \in R^{n} \quad x_{i}-y_{i} \mid<\delta, i=\right.$ $1, \ldots, n\}$. Since $\psi$ is ACL, there exists a set $F_{1} \subset F$ with $m_{n-1}\left(F \backslash F_{1}\right)$ $=0$ such that $\psi$ is absolutely continuous on every closed subsegment of $I_{u}=\left\{u+t e_{u}: 0<t \leq \delta\right\}$ whenever $u \in F_{1}$. On the other hand, by $[4,2.14,2.20,2.26]$ we have 
(5.4) $\frac{1}{K_{\boldsymbol{O}}(f)} \int_{C_{1}}\left|\psi^{\prime}(x)\right|^{n} d m_{n}(x) \leq \int_{C_{1}} J(x, \psi) d m_{n}(x) \leq \int_{R^{n}} N\left(w, \psi, C_{1}\right) d m_{n}(w)$

$$
\leq N(f, G) m_{n}\left(\psi C_{1}\right)<\infty .
$$

It follows that

$$
\int_{I_{u}}\left|\psi^{\prime}(x)\right| d m_{1}(x)<\infty
$$

for all $u$ in a set $F_{2}$ with $m_{n-1}\left(F \backslash F_{2}\right)=0$. If $u \in F_{1} \cap F_{2}$, the limit (5.3) exists. This proves the assertion. We denote by $T$ the subset of $\varphi A$ consisting of points $u$ where the limit (5.3) exists.

From the assumptions it follows that $D=G \cup_{A} \cup_{\alpha} G$ is a domain. We next define a mapping $\tilde{\psi}: q D \rightarrow \bar{R}^{n}$ by setting

$$
\begin{array}{ll}
\tilde{\psi}(x)=\psi(x) & \text { if } x \in \varphi G, \\
\tilde{\psi}(x)=\lim _{t \rightarrow 0} \psi\left(x+t_{n}\right) & \text { if } x \in T, \\
\tilde{\psi}(x)=0 & \text { if } x \in \varphi A \backslash T, \\
\tilde{\psi}(x)=\alpha(\psi(\beta(x))) & \text { if } x \in \varphi(\alpha G),
\end{array}
$$

where $\beta$ is the reflection in $\left\{x \in R^{n}, x_{n}=0\right\}$. The mapping $\tilde{\psi}$ is a (possibly discontinuous) ACL-mapping and the partial derivatives are locally $L^{n}$-integrable by (5.4). It also satisfies

$$
|\tilde{\psi}(x)|^{n} \leq K_{o}(f) J(x, \tilde{\psi}) \text { a.e. }
$$

By a result of Rešetnjak [9, Theorem 1] there exists a quasimeromorphic mapping $\hat{\psi}$ which coincides with $\tilde{\psi}$ a.e. in $\varphi D$ and $K(\hat{\psi})=K(\psi)=K(f)$. Then also $\hat{\psi}$ is an extension of $\psi$ and the required mapping is $g=$ $\hat{\psi} \circ \varphi \mid D$.

5.5. Corollary. If $f: B^{n} \rightarrow B^{n}$ is a quasiregular mapping with $C\left(f, \partial B^{n}\right)$ $\subset \partial B^{n}$, there exists a quasimenmorphic mapping $g: \bar{R}^{n} \rightarrow \bar{R}^{n}$ such that $g \mid B^{n}=f$ and $K(g)=K(f)$.

5.6. Remark. Theorem 5.2 has also been proved by F. W. Gehring.

5.7. Radial and angular limits. One of the basic classical results in the boundary behavior of analytic functions is Fatou's theorem which states that a bounded analytic function of a disc has radial limits at almost all boundary points. This is no longer true for quasiregular mappings in the case $n=2[7$, p. 119; 11]. Whether Fatou's theorem holds for quasiregular mappings in the case $n \geq 3$ is not known at present, it is even not known whether such a mapping always must have at least one radial 
A. I. 507

limit. In 5.14 we show that if the multiplicity does not increase too rapidly when approaching the boundary, then the assertion in Fatou's theorem is true. Here we show in 5.8 that the existence of a radial limit implies the existence of an angular limit.

If $y \in S^{n-1}$ and $0<\varphi<\pi / 2$, we set

$$
K(y, \varphi)=\left\{x \in R^{n}|(y \mid y-x)>| y-x \mid \cos \varphi\right\} .
$$

5.8. Theorem Let $f: B^{n} \rightarrow \bar{R}^{n}$ be a quasimeromorphic mapping with cap $\mathbf{C} f B^{n}>0$ and let $y \in \partial B^{n}$. Suppose that the radial limit $b=\lim _{t \rightarrow 1} f(t y)$ exists and assume $0<\varphi<\pi / 2$. Then also the angular limit

$$
\lim _{\substack{x \rightarrow y \\ x \in K}} f(x)
$$

exists and equals $b$.

Proof. Let $\varphi<\varphi_{0}<\pi / 2$ and set $K_{0}=K\left(y, \varphi_{0}\right) \cap B^{n}(y, 1)$. Suppose that the limit (5.9) does not exist. Then there is a sequence $\left(x_{i}\right)$ of points in $K(y, \varphi)$ converging to $y$ with $b_{1}=\lim _{i \rightarrow \infty} f\left(x_{i}\right) \neq b$. There exists $j$ such that $K_{i}=K\left(y, \varphi_{0}\right) \cap B^{n}\left(y, 2\left|y-x_{i}\right|\right) \subset B^{n} \quad$ whenever $i \geq j$. Let $h_{i}$ be the similarity mapping of $K_{0}$ onto $K_{i}$. By $[5,3.17]$ the family of mappings $g_{i}=f^{\circ} h_{i}$ is equicontinuous. Hence by $[10$. p. 664] there exists a subsequence of $\left(g_{i}\right)$ which converges to a quasimeromorphic mapping $g$. There exists a point $z$ in $\bar{K}(y, \varphi) \cap$ $S^{n-1}(y, 1 / 2) \subset K_{0}$ which is a limit of a subsequence of $\left(h_{i}^{-1}\left(x_{i}\right)\right)$. Then $g(z)=b_{1}$. But $g$ maps the whole segment $\gamma_{y} \cap K_{0}$ on the point $b$ which implies that $g$ is constant. This contradiction proves the theorem.

5.10. Asymptotic limits. We shall now prove that a quasimeromorphic mapping of a ball omitting a set of positive capacity has asymptotic limits in a dense set on the boundary.

5.11. Theorem. Let $f: B^{n} \rightarrow \bar{R}^{n}$ be a quasimeromorphic mapping with cap $\mathbf{C} f B^{n}>0$. Then for every $y$ in a dense set $Y$ in $\partial B^{n}$ there exists a path $\gamma:[0,1] \rightarrow \bar{B}^{n}$. with $\gamma[0,1) \subset B^{n}$ and $\gamma(1)=y$ such that $\lim _{t \rightarrow 1} f(\gamma(t))$ exists.

Proof. Let $z$ be a point of $\partial B^{n}$ and let $\varepsilon>0$. It suffices to show that there exists a path $\gamma:[0,1] \rightarrow \bar{B}^{n}$ with $\gamma[0,1) \subset B^{n}$ and $\gamma(1)$ $\in \partial B^{n} \cap B^{n}(z, \varepsilon)$ such that $\lim _{t \rightarrow 1} f(\gamma(t))$ exists. If the radial $\operatorname{limit} \lim _{t \rightarrow 1} f(t z)$ exists, there is nothing to prove. Suppose that $f$ has no radial limit at $z$. 
Then there is a sequence $s_{1}<s_{2}<\ldots$ of positive numbers such that $\lim _{i \rightarrow \infty} s_{i}=1$ and $\lim _{k \rightarrow \infty} f\left(\gamma_{z}\left(s_{2 k}\right)\right)=u \neq \lim _{k \rightarrow \infty} f\left(\gamma_{z}\left(s_{2 k+1}\right)\right)=v$. There exists $k_{0}$ such that for $k \geq k_{0} 1-s_{2 k}<\varepsilon$ and the spherical diameter of $F_{k}=$ $f\left(\gamma_{z}\left[s_{2 k}, s_{2 k+1}\right]\right)$ is at least a fixed positive number. Let $\Gamma_{k}$ be the family of rectifiable paths $l:[0,1] \rightarrow \bar{R}^{n}$ with $l(0) \in F_{k}$ and $l(1) \in \mathbf{C} f B^{n}$. Then by $[5,3.11]$ and $[14$, Theorem 3.8] there is a positive number $\alpha$ such that

$$
M\left(\Gamma_{k}\right) \geq \lambda
$$

for $k \geq k_{0}$. Let $\tilde{\Gamma}_{k}$ be the family of maximal liftings $[6,3.11]$ of the paths in $\Gamma_{k}$ starting at some point in $\gamma_{z}\left[s_{2 k}, s_{2 k+1}\right]$ and let $\hat{\Gamma}_{k}$ be the family of rectifiable paths in $\tilde{\Gamma}_{k}$. Every path in $\hat{\Gamma_{k}}$ is of the form $\hat{l}:[0, u) \rightarrow B^{n}$, and the limits $\lim _{t \rightarrow u} \hat{l}(t) \in \partial B^{n}$ and $\lim _{t \rightarrow u} f(\hat{l}(t))$ exist. Suppose now that every $\hat{l} \in \hat{\Gamma}_{k}$ ends outside $B^{n}(z, \varepsilon)$. Then we have the estimate

$$
M\left(\tilde{\Gamma}_{k}\right)=M\left(\hat{\Gamma}_{k}\right) \leq \prime_{n-1}\left(\log \frac{\varepsilon}{1-s_{2 k}}\right)^{1-n} .
$$

On the other hand, we have $M\left(\Gamma_{k}\right) \leq M\left(f \tilde{\Gamma}_{k}\right)$ and by (2.6) $M\left(f \tilde{\Gamma}_{k}\right) \leq$ $K_{I}(f) M\left(\tilde{\Gamma}_{k}\right)$. Combining these with $(5.12)$ and $(5.13)$ we get a contradiction as $k \rightarrow \infty$. We have proved the existence of a path $\gamma$ with the required properties.

5.14. Mappings with bounds on the increase of the multiplicity. We shall in the rest of the paper consider the class $F(c, p)$ of quasiregular mappings $f: B^{n} \rightarrow B^{n}$ with the property that for $0<r<1 \quad N\left(f, \bar{B}^{n}(r)\right) \leq$ $c(1-r)^{-p}$ where $c<\infty$ and $p, 0 \leq p<n-1$. are constants. We shall first prove Fatou's theorem for these mappings.

5.15. Theorem. Let $f: B^{n} \rightarrow B^{n}$ be a quasiregular mapping in the class $F(c, p)$ with $c<\infty$ and $0 \leq p<n-1$. Then $f$ has radial limits a.e. in $\partial B^{n}$.

Proof. Let $\alpha \in(0,1)$ be such that $2^{p-(n-1)}<x^{n}$. For $y \in \partial B^{n}$ and for any positive integer $k$ we let $\gamma_{y, k}$ be the path $\gamma_{y}:\left[1-2^{-k}, 1-2^{-k-1}\right]$, where we recall that $\gamma_{y}(t)=t y$, and define the closed set

$$
A_{k}=\left\{y \in \partial B^{n} \mid d\left(f ; \gamma_{y, k}\right) \geq \lambda^{k}\right\} .
$$

We let $\Gamma_{k}$ be the family of paths $\gamma_{y, k}, y \in A_{k}$. If

$$
\begin{aligned}
& \varrho(x)=\alpha^{-k} \text { for } x \in B^{n}, \\
& \varrho(x)=0 \text { elsewhere, }
\end{aligned}
$$


then

$$
\int_{\gamma} \varrho d s \geq 1
$$

for every $\gamma \in f \Gamma_{k}$. Hence

$$
M\left(f \Gamma_{k}\right) \leq \Omega_{n} x^{-k n} .
$$

On the other hand,

$$
M\left(\Gamma_{k}\right)=m_{n-1}\left(A_{k}\right)\left(\log \frac{1-2^{-k-1}}{1-2^{-k}}\right)^{1-n} \geq m_{n-1}\left(A_{k}\right) 2^{k(n-1)} .
$$

Since $f \in F(c, p)$, we get by $[4,3.2]$

$$
M\left(\Gamma_{k}\right) \leq K_{o}(f) N\left(f, \bar{B}^{n}\left(1-2^{-k-1}\right)\right) M\left(f \Gamma_{k}\right) \leq K_{o}(f) c 2^{(k+1) p} \Omega_{n} \alpha^{-k n} .
$$

Combining this with $(5.16)$ we get

$$
\begin{aligned}
& m_{n-1}\left(\bigcup_{k=q}^{\infty} A_{k}\right) \leq \Omega_{n} K_{O}(f) c 2^{p} \sum_{k=q}^{\infty}\left(2^{p-(n-1)} \alpha^{-n}\right)^{k} \\
& \leq 2^{p} \Omega_{n} K_{O}(f) c \beta^{q} /(1-\beta) \rightarrow 0 \text { as } q \rightarrow \infty,
\end{aligned}
$$

where $\beta=2^{p-(n-1)} \alpha^{-n}<1$. If $q \geq 1$ is any integer and

the radial limit

$$
y \in \mathbf{C} \bigcup_{k=q}^{\infty} A_{k}
$$

$$
\lim _{t \rightarrow 1} f(t y)
$$

exists by the definition of $A_{k}$ and the fact that $\alpha<1$. The theorem is proved.

Our second result for mappings in the class $F(c, p)$ is known as the theorem of $\mathrm{F}$. and $\mathrm{M}$. Riesz for analytic mappings.

5.17. Theorem. Let $f: B^{n} \rightarrow B^{n}$ be a quasiregular mapping in the class $F(c, p)$ with $c<s$ and $0 \leq p<n-1$. Then there exists no measurable set $A \subset \partial B^{n}$ with $m_{n-1}(A)>0$ such that the radial limit $\lim _{t \rightarrow 1} f(t y)$ equals a constant $b$ for all $y \in A$.

Proof. Suppose that there is such a set A. There exists $\lambda, 1 / 2 \leq \lambda<1$, such that $z=\lambda e_{1}, f(z)=z^{\prime} \neq b,-e_{1} \in A$, and $m_{n-1}(E)>0$ for some $\varphi \in(0, \pi / 2)$ where $E=A \cap C$ and

$$
C=\left\{x \in R^{n},\left(x ;-e_{1}\right)>|x| \cos \varphi\right\} .
$$

Let $l:(-1, \lambda] \rightarrow B^{n}$ be the path defined by $l(t)=t e_{1}$. Then $l^{\prime}=f \circ l$ 
is a path joining the points $z^{\prime}$ and $b$. Let $t_{0} \in[0, \lambda)$ be a point such that $l^{\prime}\left[t_{0}, \lambda\right] \subset B^{n}\left(z^{\prime}, s\right)$ where $s=\left|z^{\prime}-b\right| / 2$.

Let $\pi: \bar{B}^{n} \backslash\{0\} \rightarrow S^{n-1}$ be the radial projection. We choose a number $\delta$ such that $0<\delta<1 / 2$ and $\delta^{n-1-p}<1 / 3$. For any $\varrho \in(0, s)$ and any integer $k \geq 0$ we set

$$
D(k, \varrho)=F_{\varrho} \cup C \cup S^{n-1}\left(r_{k}\right)
$$

and $E(k, \varrho)=\pi D(k, \varrho), \quad$ where $\quad F_{\varrho}=f^{-1} \bar{B}^{n}(b, \varrho) \quad$ and $\quad r_{k}=1-$ $\delta^{k}(1-\lambda)$. Let $\Gamma(0, \varrho)$ be the family of paths in $\bar{B}^{n}(\lambda)$ which join $L=$ $l\left[t_{0}, \lambda\right]$ and $D(0, \varrho)$. Then

$$
M(\Gamma(0, \varrho)) \geq \alpha(n, d(L)) m_{n-1}(E(C, \varrho)),
$$

where $\alpha(n, d(L))>0$ is a constant which depends only on $n$ and $d(L)$. On the other hand,

$$
M(f \Gamma(0, \varrho)) \leq \omega_{n-1}\left(\log \frac{s}{\varrho}\right)^{1-n} .
$$

By $[4,3.2]$

$$
M(\Gamma(0, \varrho)) \leq K_{o}(f) N\left(f, \bar{B}^{n}(\hat{\lambda})\right) M(f \Gamma(0, \varrho)) .
$$

Combining $(5.18)-(5.20)$ we may choose $\varrho_{0} \in(0 . s)$ such that

$$
m_{n-1}\left(E\left(0, \varrho_{0}\right)\right)<m_{n-1}(E) / 4 \text {. }
$$

For $y \in S^{n-1}$ and $k \geq 1$ we define the path $\gamma_{y, k}=\gamma_{y} \mid\left\lfloor r_{k-1}, r_{k}\right]$ (see 2.1).

We will now choose a sequence $\varrho_{1}, \varrho_{2} \ldots$ of positive numbers with $\varrho_{0}>\varrho_{1}>\varrho_{2}>\ldots$ inductively as follows. Suppose that $\varrho_{k-1}$ is chosen, $k \geq 1$. For $\varrho \in\left(0, \varrho_{k-1}\right)$ we set

$$
\Gamma(k, \varrho)=\left\{\gamma_{y, k} \mid y \in E(k, \varrho) \backslash \bigcup_{i=0}^{k-1} E\left(i, \varrho_{i}\right)\right\} .
$$

Then

$$
\begin{gathered}
M(\Gamma(k, \varrho))=m_{n-1}\left(E(k, \varrho) \backslash \bigcup_{i=0}^{k-1} E\left(i, \varrho_{i}\right)\right)\left(\log \frac{r_{k}}{r_{k-1}}\right)^{1-n} \\
\geq m_{n-1}\left(E(k, \varrho) \backslash \bigcup_{i=0}^{k-1} E\left(i, \varrho_{i}\right)\right) \delta^{(k-1)(1-n)} .
\end{gathered}
$$

By the definition of the sets $E(k, \varrho)$ every path in $f \Gamma(k, \varrho)$ joins the sets $\bar{B}^{n}(b, \varrho)$ and $\mathbf{C} \bar{B}^{n}\left(b, \varrho_{k-1}\right)$, hence

$$
M(f \Gamma(k, \varrho)) \leq \omega_{n-1}\left(\log \frac{\varrho_{k-1}}{\varrho}\right)^{1-n} .
$$


Since $f$ is in the class $F(c, p)$, we have by $[4,3.2]$

$$
M(\Gamma(k, \varrho)) \leq K_{o}(f) c\left(1-r_{k}\right)^{-p} M(f \Gamma(k, \varrho)) .
$$

Combining $(5.21)-(5.23)$ we obtain

$$
\begin{gathered}
m_{n-1}\left(E(k, \varrho) \backslash \bigcup_{i=0}^{k-1} E\left(i, \varrho_{i}\right)\right) \\
\leq \omega_{n-1} K_{o}(f) c(1-\lambda)^{-p} \delta^{-p} \delta^{(n-1-p)(k-1)}\left(\log \frac{\varrho_{k-1}}{\varrho}\right)^{1-n} .
\end{gathered}
$$

We choose $\varrho=\varrho_{k}$ such that the right hand side of (5.24) equals $m_{n-1}(E) / 2^{k+2}$.

It follows that

$$
\frac{\varrho_{k-1}}{\varrho_{k}} \leq \exp \left(x\left(\frac{2}{3}\right)^{(k-1)(n-1)}\right), k=1,2, \ldots,
$$

where $\alpha$ is a positive constant which does not depend on $k$. From $(5.25)$ we get

$$
\lim _{k \rightarrow \infty} \varrho_{k} \geq \varrho_{0}\left[\exp \left(\alpha /\left(1-\left(\frac{2}{3}\right)^{1 /(n-1)}\right)\right)\right]^{-1}>0 .
$$

On the other hand,

$$
m_{n-1}\left(\bigcup_{k=0}^{\infty} E\left(k, \varrho_{k}\right)\right) \leq m_{n-1}(E) / 2
$$

by $(5.24)$ and by the choic of $\varrho_{k}, k=0,1,2, \ldots$ Hence we may choose

$$
y \in E \backslash \bigcup_{k=0}^{\infty} E\left(k, g_{k}\right) \text {. }
$$

By the definition of the sets $E\left(k, \varrho_{k}\right)$ we have $f\left(r_{k} y\right) \notin \bar{B}^{n}\left(b, \varrho_{k}\right)$. But $b=\lim _{t \rightarrow 1} f(t y)$ which contradicts $(5.26)$. The theorem is proved.

5.27. Remark. It follows from [6, 2.3] that for a quasiregular mapping $f: B^{n} \rightarrow B^{n}, n \geq 3$, which is a local homeomorphism we have $N\left(f, \bar{B}^{n}(1-\delta(1-\mathrm{r})) \backslash B^{n}(r)\right) \leq c(1-r)^{1-n}$, where $0<\delta<1$ and $c<\infty$. The proofs of 5.15 and 5.17 can be carried through by the use of this type of estimate with $1-n$ replaced by $-p, 0 \leq p<n-1$. This makes the question whether the statements in 5.15 and 5.17 hold for local homeomorphisms in the case $n \geq 3$ rather interesting.

University of Helsinki

Helsinki, Finland 


\section{References}

1. Agard, S. and A. Marden: A removable singularity theorem for local homeomorphisms. - Indiana Math. J. 20 (1970), 455-461.

2. Cartwright, M. L.: Integral functions. - Cambridge University Press, 1962.

3. Collingwood, E. F. and A. J. Lohwater: The theory of cluster sets. - Cambridge University Press, 1966.

4. Martio, O., S. Rrckman, and J. Värsälä: Definitions for quasiregular mappings. - Ann. Acad. Sci. Fenn. A I 448 (1969), 1-40.

5. - - Distortion and singularities of quasiregular mappings. - Ann. Acad. Sci. Fenn. A I 465 (1970), 1-13.

6. - - Topological and metric properties of quasiregular mappings. - Ann. Acad. Sci. Fenn. A I 488 (1971), I--31.

7. Noshiro, K.: Cluster sets. - Springer Verlag, 1960.

8. Роцескі, Е.А. (Полецьий, Е. А.): Метод модулей дпя негомеоморфных квазиконформных отображений. - Mat. Sbornik 83 (1970), $261-272$.

9. Rešetnjak, J. G. (Решетняк. Ю. Г.): Оценкі уодуля непрерывности для некоторых отображений. - Sibirsk. Mat. Z̆. 7 (1966), 1106-1114.

10. - - - Отображения с ограниченным пскажением как экстремали интегралов типа Дiгрихте. - Sibirsk. Mat. Z̆. 9. (1968), 652-666.

11. Storvick, D. A.: Radial limits of quasiconformal functions. - Nagoya Math. J. 23 (1963), $199-206$.

12. Wallin, H.: A connection between $\alpha$-capacity and $L^{p}$-classes of differentiable functions. - Arkiv för Mathematik 5, (1964), 331-341.

13. VÄISÄLÄ, J.: Lectures on $n$-dimensional quasiconformal mappings. - Lecture Notes in Mathematics 229, Springer Verlag, 1971.

14. Ziemer, W. P.: Extremal length and p-capacity. - Michigan Math. J. 16 (1969), $43-51$.

15. Zorič, V. А. (Зорич, В. А.): Теорема М. А. Ааврентьева о квазиконформных отображениях пространства. - Mat. Sbornik 74 (1967), 417-433.

16. - - Изолированная особенность отображений с огранцченным пскажением. Mat. Sbornik 81 (1970), $634-638$. 